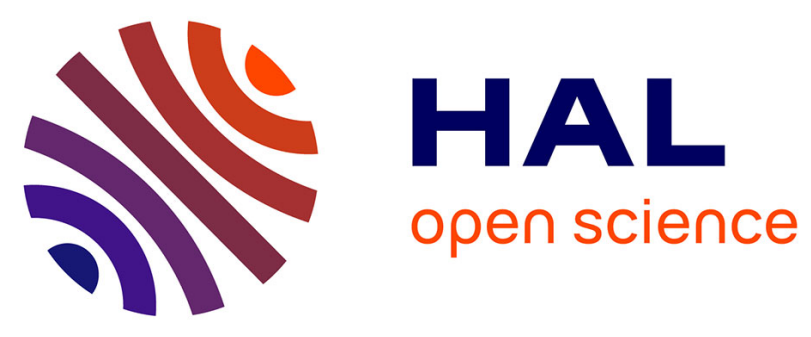

\title{
Effect of Cumyl Alcohol in Multilayer Dielectric on Space Charge Build Up
}

Benny Reinmart, G. Teyssedre, Séverine Le Roy, Ngapuli Sinisuka

\section{To cite this version:}

Benny Reinmart, G. Teyssedre, Séverine Le Roy, Ngapuli Sinisuka. Effect of Cumyl Alcohol in Multilayer Dielectric on Space Charge Build Up. 2019 IEEE 20th International Conference on Dielectric Liquids (ICDL), Jun 2019, Roma, France. pp.1-4, 10.1109/ICDL.2019.8796775 . hal-02340312

\section{HAL Id: hal-02340312 \\ https://hal.science/hal-02340312}

Submitted on 17 Nov 2020

HAL is a multi-disciplinary open access archive for the deposit and dissemination of scientific research documents, whether they are published or not. The documents may come from teaching and research institutions in France or abroad, or from public or private research centers.
L'archive ouverte pluridisciplinaire HAL, est destinée au dépôt et à la diffusion de documents scientifiques de niveau recherche, publiés ou non, émanant des établissements d'enseignement et de recherche français ou étrangers, des laboratoires publics ou privés. 


\section{Effect of Cumyl Alcohol in Multilayer Dielectric on Space Charge Build Up}

\author{
Benny Reinmart \\ Technology Development and Standardization \\ PT. PLN (Persero) \\ Jakarta, Indonesia \\ benny.reinmart@gmail.com \\ Séverine Le Roy \\ LAPLACE, UMR 5213 \\ University of Toulouse (CNRS, INPT, UPS) \\ Toulouse, France \\ severine.leroy@laplace.univ-tlse.fr
}

\begin{abstract}
One of the issues of using cross-linked Polyethylene (XLPE) in DC insulation systems is the propensity of crosslinking byproducts to promote charge buildup inside the insulation, leading potentially to insulation failure. Field-induced ionization of the molecules, like in insulating liquids, is thought to be one of the processes of charge generation. In this paper, space charge measurement is realized to probe the effect of cumyl alcohol as one of the crosslinking byproducts. Low Density Polyethylene (LDPE) is used as polymer matrix, and soaked in cumyl alcohol. We show that polyethylene naphthalate (PEN) layers constitute efficient barriers to the evaporation of cumyl alcohol from LDPE films. The space charge behavior of soaked LDPE is compared to that of reference LDPE with PEN. Significant charge build-up occurs at the LDPE/PEN interfaces due to the conductivity gradient. PEN appears not suited for such analysis as the field is concentrated in the layer and is weak in the bulk LDPE.
\end{abstract}

Keywords-space charge; crosslinking byproducts; cumyl alcohol; low density polyethylene.

\section{INTRODUCTION}

Crosslinked Polyethylene (XLPE) is used for long as insulation in High Voltage AC systems, particularly cables. XLPE has better thermomechanical stability, lasts longer, and shows less moisture sensitivity compared to LDPE [1]. Despite those advantages, there is some weakness in XLPE due to the effect of crosslinking byproducts and these effects are detrimental to the use of XLPE for HVDC systems.

During the cross-linking process, peroxides decompose to create free radicals that can initiate chemical bonding between polymeric chains. This process forms byproducts such as dimethyl benzyl alcohol (cumyl alcohol), acetophenone, and $\alpha$-methylstyrene. These byproducts remain in the insulation and migrate out slowly during the time and affect the electrical properties of the material. Conductivity is generally increased in presence of byproducts, but the underlying mechanisms are not agreed upon. Three processes can be envisaged, being injectionassisted process for electronic carriers [2], transport-assisted process for these charges [3], and promotion of ionic transport either by direct ionization under field or indirectly through ion solvation effects [4].

Byproducts can also cause deep chemical traps in XLPE, i.e. centers that stabilize electrical charges as it is probed with photoluminescence techniques [5]. Hirai et al. show that byproducts increase the conductivity, but only cumyl alcohol creates carrier traps [6].

\author{
Gilbert Teyssedre \\ LAPLACE, UMR 5213 \\ University of Toulouse (CNRS, INPT, UPS) \\ Toulouse, France \\ gilbert.teyssedre@laplace.univ-tlse.fr \\ Ngapuli I. Sinisuka \\ School of Electrical Engineering and Informatics \\ Bandung Institute of Technology \\ Bandung, Indonesia \\ n_sinisuka@yahoo.com
}

Our purpose in this work is to investigate possible ionization effects associated with crosslinking byproducts. Though the matrix is a solid, this has relation to dielectric liquids, as ionization processes appear to be much better understood in liquids than in solids, with as common driving parameter the ionization potential of molecules [7] to explain streamer propagations in liquids [8] and resistance to treeing processes in solids $[9,10]$ for example. The other link to dielectric liquids is the need to maintain the liquid additive into the matrix and control the product uptake.

In this work, cumyl alcohol is chosen as investigated byproduct. It is incorporated into Low Density Polyethylene (LDPE) as a base material of XLPE. The tests consist of space charge measurement using Pulse Electro-Acoustic (PEA) method and conduction current measurements. To keep the cumyl alcohol amount as much controlled as possible inside the specimen for several hours during the electrical characterization, films of Polyethylene Naphthalate (PEN) are intercalated at both faces of LDPE samples. PEN was chosen since available in relatively thin form and for its good barrier properties. This constitutes an originality of the work carried out. Though the purpose of the measurement is not to investigate in particular PEN properties, its impact on the results must be considered.

\section{SAMPLES PREPARATION}

\section{A. Materials}

The LDPE samples used in these measurements have an average thickness of $340 \mu \mathrm{m}$ and diameter of $8 \mathrm{~cm}$. They were obtained by press-molding technique at $150^{\circ} \mathrm{C}$ from LDPE pellets. Polyimide layers were used to ease the sample removal after the molding process is completed. Polyimide layer is chosen because it does not affect the LDPE sample with decomposition products when it is processed at high temperature. To approach the desired thickness, an epoxy spacer with $350 \mu \mathrm{m}$ thickness is selected.

PEN films, 12 or $25 \mu \mathrm{m}$ thick, were cut into circular shape following the shape of LDPE sample. Gold metallization is done on one face of each PEN layer. The purpose of the metallization is to have same electrode conditions on each side of the sample. Gold electrodes were deposited by cold sputtering with diameter of $2 \mathrm{~cm}$ and a thickness of $50 \mathrm{~nm}$. Thus, the PEN film will be placed in upper and bottom side of the LDPE sample. This structure is used in each measurement as multi-layered dielectric. The illustration of the sample can be seen in Fig. 1. 


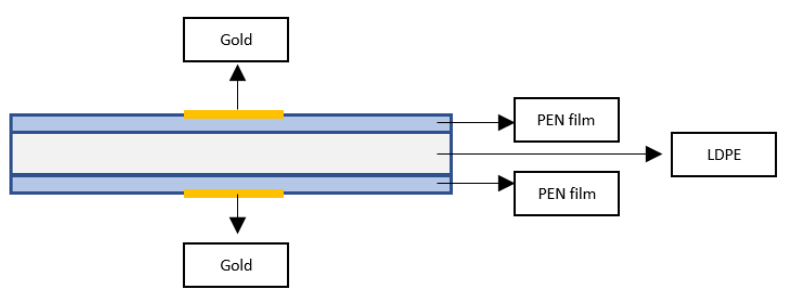

Fig. 1. Illustration of multilayered dielectric for the measurement

\section{B. Cumyl Alcohol uptake}

The first measurement is to observe the PEN barrier effect by comparing samples soaked with and without PEN for up to 5 hours at $70^{\circ} \mathrm{C}$. The absorption rate is taken every 30 minutes, and before the sample is put in balance to weight, it is rinsed with acetone, ethanol, and deionised water to remove cumyl alcohol from the surface of the sample. Fig. 2a shows that after 5 hours both samples have a same cumyl alcohol percentage $(5.34 \%$ and $5.35 \%)$.

The evaporation rate is an important characteristic as it controls the amount of chemical during electrical measurements. For the evaporation rate, the weighing process is repeated in intervals of $20 \mathrm{~min}$ for the first 4 hours after the dipping process, and the data is continually recorded until 18 hours after the dipping process. Both samples are weighed at room temperature. Sample 1 is weighed without PEN layers, while sample 2 is weighed with $12 \mu \mathrm{m}$ thick PEN layers on the entire surface.

To make sure that the cumyl alcohol is not absorbed by

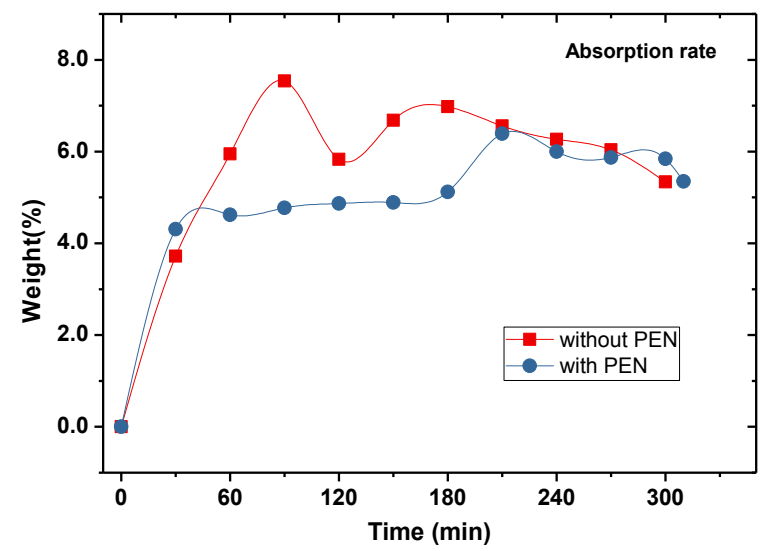

(a)

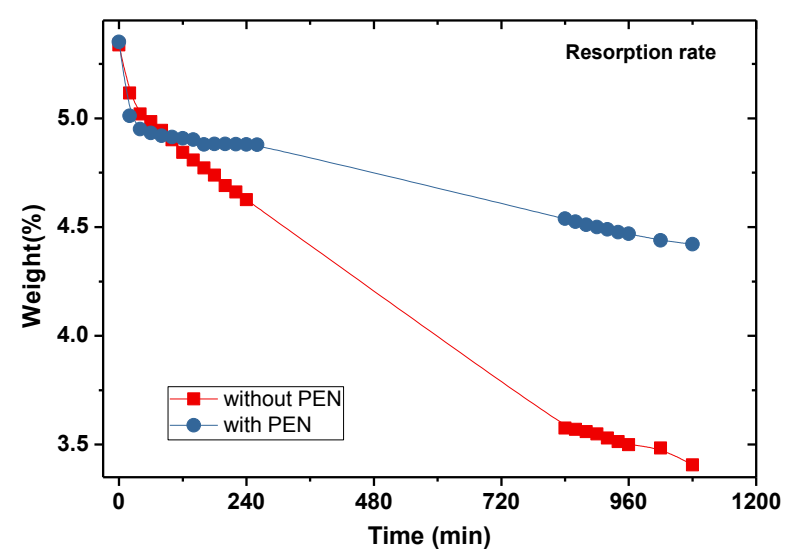

(b)

Fig. 2. Cumyl alcohol absorption rate (a) and evaporation rate (b) vs time. The evaporation rate was measured after dipping for $5 \mathrm{~h}$.
PEN layers, the later were weighed before and after the measurement process and no weight variation was detected. Based on Fig. 2.b, which shows that after 18 hours, the percentage of cumyl alcohol in sample 2 is substantially higher than in sample 1, quantitatively it can be stated that the effect of PEN layers as a barrier to cumyl alcohol evaporation is effective. Thus, this weight measurement will be done after each space charge and conduction current measurement.

\section{ELECTRICAL MEASUREMENTS}

\section{A. Protocol}

Space charge measurements have been performed in different configurations of sample such as:

- Untreated LDPE only

- Untreated LDPE with $12 \mu \mathrm{m}$ PEN layers

- Untreated LDPE with $25 \mu \mathrm{m}$ PEN layers

- LDPE soaked in cumyl alcohol for $4 \mathrm{~h}$ with $12 \mu \mathrm{m}$ PEN layers

The same protocol, depicted in Fig. 3, was applied for every sample configuration with $30 \mathrm{~min}$ polarization and 30 min depolarization. The applied field is from 5 to $50 \mathrm{kV} / \mathrm{mm}$ for positive polarity and then -5 to $-50 \mathrm{kV} / \mathrm{mm}$ for negative polarity. Considering the presence of cumyl alcohol, all the space charge measurements were taken at $25^{\circ} \mathrm{C}$ to prevent any evaporation. The temperature is controlled automatically in the oven chamber. Charge profiles were recorded all along the protocol in $30 \mathrm{~min}$ intervals.

\section{B. Space charge measurement results}

Fig. 4 shows space charge results obtained for the different materials. Data for $25 \mu \mathrm{m}$ thick PEN layers are not shown for sake of space saving.

a) Untreated LDPE: The space charge pattern obtained in LDPE alone is plotted in Fig. 4a. It constitutes the reference for the results. LDPE was not provided with gold electrodes. From the cartography, positive charge dominates in the insulation, consistently with previous results obtained on the same material. The charge density inside the bulk increases with the increase of applied voltage on the sample. After polarity reversal a significant amount of negative charges is detected. Presumably, the positive charge stored in the first run increases the field at the electrodes and triggers negative charge injection.

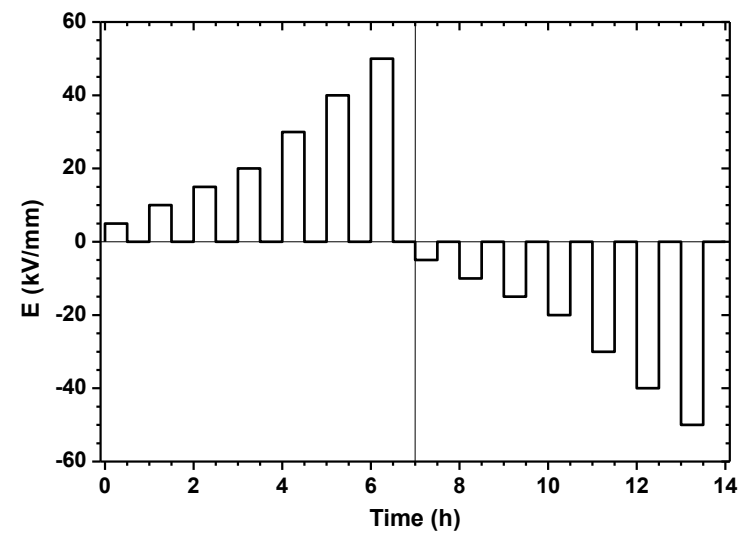

Fig. 3. Space charge measurement protocol 
b) Untreated LDPE with PEN layers. Fig. 4b shows the charge pattern when $12 \mu \mathrm{m}$ thick gold-metallized PEN layers are interposed at both sides of the LDPE. LDPE is not soaked with cumyl alcohol in this case, to see the effect of PEN layers on space charge behavior. At time 4-5 h a positive heterocharge builds up from $30 \mathrm{kV} / \mathrm{mm}$ near the ground electrode (bottom) and stays there until the applied field is $-30 \mathrm{kV} / \mathrm{mm}$. The charge distribution inside the bulk is having a periodic repetition of plans of charges with alternated polarity. The (positive) charge build up occurs at the region $0-25 \mu \mathrm{m}$ which corresponds to the region of PEN layers in the sample. It can be assumed that the charges build up is due to the existence of PEN layers on the sample. In positive polarity especially at low field, there is no heterocharges detected. To distinguish the effect of the PEN layer, space charge measurements were achieved with $25 \mu \mathrm{m}$ thick layers. The pattern (not shown) is qualitatively similar to that of Fig. $4 \mathrm{~b}$ but the charge amount is substantially less. The positive heterocharges build up appear at an applied field of $30 \mathrm{kV} / \mathrm{mm}$. The charge inside the bulk which comes periodically with different orientation polarization is still present but this time in the region 0$50 \mu \mathrm{m}$. The period of oscillations is also multiplied by 2 .

The space charges found in this configuration is rather complex and may have several origins: i) orientation polarisation of PEN, which is a polar material [11]; ii) charges migrating into LDPE and being blocked at the interface with PEN; iii) charges injected in the PEN layer; iv) interface charge build-up due to the difference in permittivity of the two materials. Finally, because of mismatch of acoustic properties of the materials, multiple reflection in the PEN layers may produce apparent acoustic signal in the bulk. The periodic nature of the charge found in the buk is certainly associated with this later process. The period of oscillations is about twice for the thicker PEN because the waves need more time to be reflected. Charge injection from PEN is not obvious since it would provide homocharge rather than heterocharge. An interfacial charge (Maxwell-Wagner-Sillars effect) [12] is certainly present, but it does not explain all the feaures: the charge seems clearly stabilized at the LDPE/PEN interface and needs strong reversed field to be modified. A similar process seems to be at play at the high voltage (top) electrode.

c) LDPE soaked in cumyl alcohol with PEN layers. The space charge pattern for soaked LDPE with $12 \mu \mathrm{m}$ thick PEN layers is shown in Fig. 4.c. The measurement takes 15 hours time to be completed. The sample was weighed before soaked in cumyl alcohol, after soaked and after doing space charge measurement. After 15 hours measurement, there is still about $3.7 \%$ cumyl alcohol inside the sample, vs. $5.7 \%$ initially, which is consistent with Fig. 2 b.

Comparatively to the case of Fig 4.b there is clearly much more charges. Already after the first step at $5 \mathrm{kV} / \mathrm{mm}$, heterocharges settle near both electrodes. For the highest fields applied, there are two prominent peaks at each electrodes and it is really difficult to localize the dielectric/dielectric interface. In the $3^{\text {rd }}$ to $5^{\text {th }}$ voltage steps, there seems to be negative charge injection combined to heterocharge build up. The bulk of the insulation shows periodic charge patterns, dominated by positive charges in the first half of the cycle and by negative charges in the end of the second half: charges seem to be a repetition of the heterocharge.

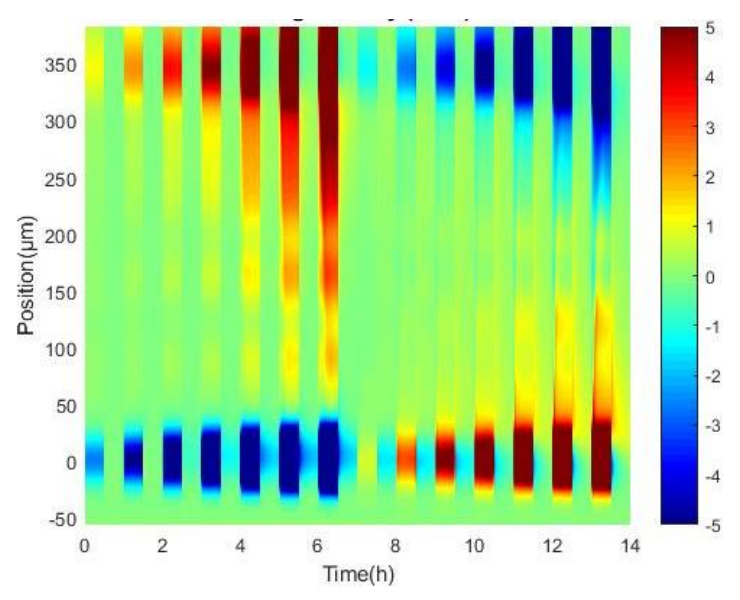

(a) untreated LDPE sample without PEN layer

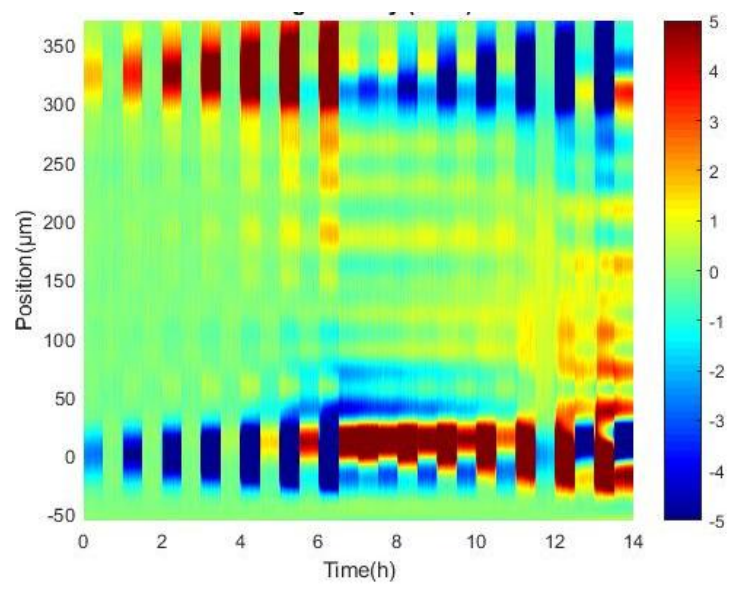

(b) untreated LDPE sample with $12 \mu \mathrm{m}$ PEN layers

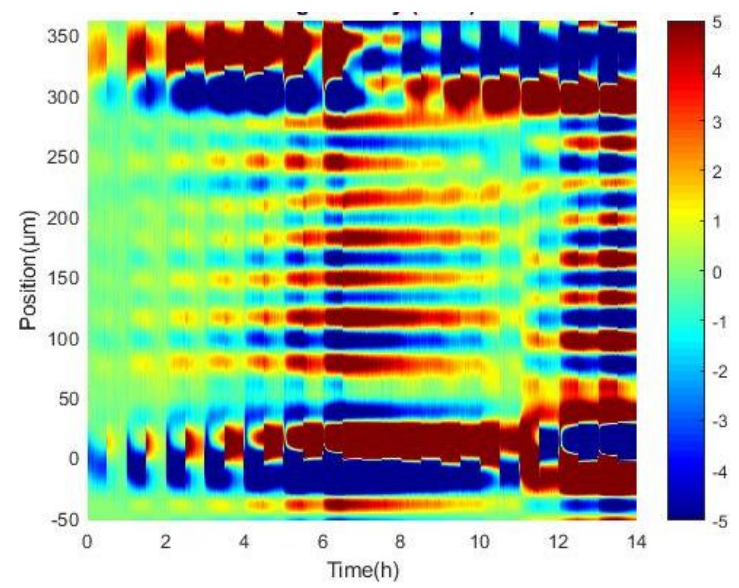

(c) LDPE sample soaked in cumyl alcohol with $12 \mu \mathrm{m}$ PEN layer

Fig. 4. Space charge distributions obtained in the different samples. The color bar represents charge density scale in $\mathrm{C} / \mathrm{m}^{3}$.

\section{Conduction current measurement}

For conduction current measurement, new samples with the same thickness $(340 \mu \mathrm{m})$ and the same diameter are used. The sample configuration for conduction current measurement is the same as for space charge measurement (PEN layers with $2 \mathrm{~cm}$ diameter gold electrodes). The temperature for this measurement is $25^{\circ} \mathrm{C}$ with the applied electrical field from $5 \mathrm{kV} / \mathrm{mm}$ to $40 \mathrm{kV} / \mathrm{mm}$. Each measurement took 30 minutes for polarisation and for depolarisation at each of the 5 field steps. The current was acquired every 2 seconds all over the cycle. 
The results from current measurements are shown in Fig. 5. From Fig. 5a, it is obvious that the sample soaked in cumyl alcohol has much more current than the reference LDPE. The current difference is almost two decades for each applied field. For $40 \mathrm{kV} / \mathrm{mm}$, the current in the sample seems to increase along with time in the two cases. This phenomenon has already been observed in LDPE and XLPE especially when stressing under high field [13].

Fig. 5.b shows the apparent conductivity vs. electrical field in the sample. Apparent means that we do not take into account the field distribution in the different layers; the field is supposed homogeneous. The difference in apparent conductivity between soaked and unsoaked sample is by two orders of magnitude. This obviously affects the electrical field distribution under DC voltage. From Figure 5.b, gross value of the conductivity of LDPE without cumyl alcohol is $10^{-15} \mathrm{~S} / \mathrm{m}$. For the sample with cumyl alcohol it is $10^{-13} \mathrm{~S} / \mathrm{m}$ as obtained for a field of $30 \mathrm{kV} / \mathrm{mm}$. Considering these values and value of the conductivity for PEN of $2.6 \times 10^{-17}$ $\mathrm{S} / \mathrm{m}$ [11], it can be anticipated that the field is contained essentially in the PEN layers, and is very high $(300 \mathrm{kV} / \mathrm{mm}$ or more) even for the unsoaked LDPE. This is consistent with the large interface charge found at the PEN/LDPE interface. The use of a less resistive layer material than PEN is necessary to investigate charging effects in the bulk.

\section{CONCLUSION}

The PEN layers tend to prevent charge injection in LDPE, but produces significant interfacial charges between dielectric layers due to differences in conductivity. Periodic charge peaks appearing inside the bulk is assumed as originating from multiple reflections of acoustic waves in the PEN layer due to acoustic impedance mismatch with LDPE. PEN is preventing the cumyl alcohol to evaporate and permits to make measurements for several hours with keeping a reasonable amount of product. Cumyl alcohol has an impact in terms of space charge build up. The presence of cumyl alcohol in the sample creates a strong heterocharge build-up near both electrodes. The LDPE conductivity is substantially enhanced when introducing cumyl alcohol, which explains the increase of interfacial charge between PEN and LDPE. Space charge results show that this charge tends to be persistent and high field is necessary to reverse it: it means that ions migrate and/or deep traps are present at the interface. Ions generated by the cumyl alcohol into the sample could produce charge accumulation and make the sample more conductive. Because PEN is a high resistivity material, the field tends to be concentrated in this layer and the configuration is not really suited to investigate field induced dissociation in the bulk material.

\section{REFERENCES}

[1] O. Peake, "The history of high voltage direct current transmission," 3rd Australas. Eng. Herit. Conf. 2009, vol. 8, no. 1, pp. 1-8, 2009.

[2] Y. Li, M. Yasuda, and T. Takada, "Influence on spatial charge distribution of cross-linking agent residues in XLPE", Proc. Int'1 Conf. Prop. Appl. Dielectr. Mater. (ICPADM), pp. 1210-1213, 1991.

[3] N. Hussin and G. Chen, "The effect of acetophenone and alphamethylstyrene on the space charge properties of low density polyethylene," Proc. IEEE Conf. Electr. Insul. Dielectr. Phenom., (CEIDP), pp. 702-705, 2008.

[4] K. Kobayashi, T. Ohara, Y. Ohki, and T. Maeno, "Effect of acetophenone on the space charge evolution in LDPE and LLDPE," Proc. IEEE Conf. Electr. Insul. Dielectr. Phenom. (CEIDP), pp. 27-31, 1999.

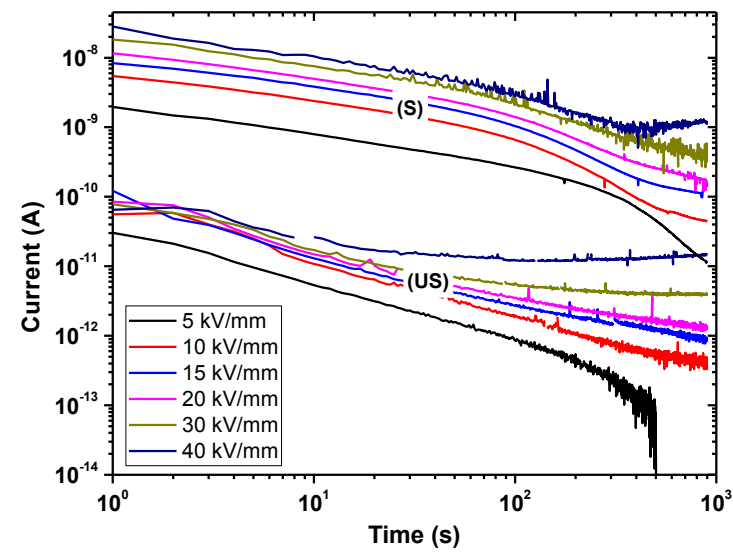

(a) Transient charging current

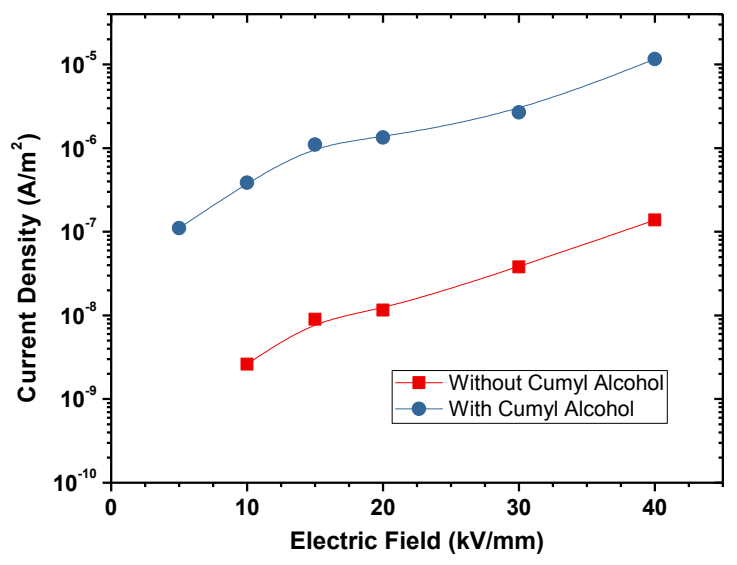

(b)Current vs. field at $1000 \mathrm{~s}$

Fig. 5. Current measurements for cumyl alcohol soaked (S) and unsoaked (US) LDPE. Both are with $12 \mu \mathrm{m}$ thick PEN layers.

[5] G. Tardieu, G. Teyssedre, and C. Laurent, "Role of additives as recombination centres in polyethylene materials as probed by luminescence techniques," J. Phys. D. Appl. Phys., vol. 35, pp. 40-47, 2002.

[6] N. Hirai, R. Minami, K. Shibata, Y. Ohki, M. Okashita, and T. Maeno, "Effect of byproducts of dicumyl peroxide on space charge formation in low-density polyethylene," Proc. Conf. Electr. Insul. Dielectr. Phenom. (CEIDP), pp. 478-483, 2001.

[7] H.S. Smalø, Ø. Hestad, S. Ingebrigtsen, and P.O. Åstrand, "Field dependence on the molecular ionization potential and excitation energies compared to conductivity models for insulation materials at high electric fields", J. Appl. Phys., vol. 109, nº 073306, 2011.

[8] N. Davari, P.-O. Åstrand, M. Unge, L. E. Lundgaard, and D. Linhjell, "Field-dependent molecular ionization and excitation energies: Implications for electrically insulating liquids", AIP Advances, vol. 4, $\mathrm{n}^{\circ} 037117,2014$.

[9] A.C. Ashcraft, R.M. Eichhorn, and R.G. Shaw, "Laboratory studies of treeing in solid dielectrics and voltage stabilization of polyethylene", Proc. IEEE International Symp. Electr. Insul., pp. 213-218, 1978.

[10] V. Englund, R. Huuva, S.M. Gubanski, and T. Hjertberg, "High efficiency voltage stabilizers for XLPE cable insulation", Polymer Degrad. Stab., vol. 94, pp. 823-833, 2009.

[11] M. Q. Hoang, L. Boudou, S. Le Roy, and G. Teyssedre, "Electrical characterization of PEN films using TSDC and PEA measurements," Proc. IEEE Int. Conf. Solid Dielectr. (ICSD), pp. 488-491, 2013.

[12] T.T.N. Vu, G. Teyssedre, B. Vissouvanadin, S. Le Roy, and C. Laurent, "Correlating conductivity and space charge measurements in multi-dielectrics under various electrical and thermal stresses", IEEE Trans. Dielectr. Electr. Insul., vol. 22, pp. 117-127, 2015.

[13] G. Teyssedre, C. Laurent, G.C. Montanari, F. Palmieri, A. See, L.A. Dissado, and J.C. Fothergill, "Charge distribution and electroluminescence in cross-linked polyethylene under dc field," J. Phys. D. Appl. Phys., vol. 34 pp. 2830-2844, 2001. 